\title{
Tendencias de morbilidad y mortalidad por neumonía en adultos mexicanos (1984-2010)
}

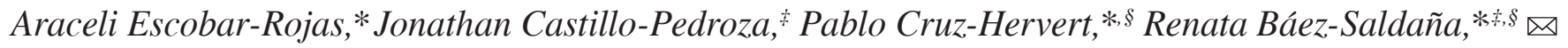

"Universidad Nacional Autónoma de México; ${ }^{\ddagger}$ Instituto Nacional de Enfermedades Respiratorias Ismael Cosío Villegas;

Instituto Nacional de Salud Pública.

Trabajo recibido: 26-I-2015; aceptado: 26-II-2015

\begin{abstract}
RESUMEN. Antecedentes: En México, los estudios de epidemiología descriptiva sobre neumonía en adultos son escasos. El objetivo fue describir en población mexicana, las tendencias de morbilidad y mortalidad nacional para neumonía en adultos de 1984 a 2010 . Métodos: Estudio ecológico de series de tiempo retrospectivo para neumonía, de 1984 a 2010, utilizando la información nacional disponible. Se midió el porcentaje de cambio del número de casos y tasa cruda y ajustada por edad. La significancia estadística de las tendencias anuales se evaluó con la prueba de tendencias no paramétrica para grupos ordenados. Resultados: La neumonía afectó principalmente a niños de 0-4 años y mayores de 65 años de edad. La morbilidad mostró una tendencia ascendente significativa. Las defunciones se mantuvieron sin observarse tendencia significativa; el análisis por grupo de edad resaltó tendencia descendente en todos los grupos, excepto en el de 15 a 64 años que observó una tendencia ascendente. Conclusiones: La morbilidad en ascenso y la mortalidad general que no observó alguna tendencia, hacen suponer que las intervenciones para mejorar el control de la neumonía aún son insuficientes. Es fundamental desarrollar investigación en la población mexicana, para identificar las áreas de debilidad y políticas de salud necesarias para observar un cambio favorable.
\end{abstract}

Palabras clave: Neumonía, México, morbilidad, mortalidad, tendencias.

ABSTRACT. Objective: To describe in Mexican population, trends in national morbidity and mortality for pneumonia in adults from 1984 to 2010. Methods: An ecological study of retrospective time series from 1984 to 2010 for morbidity, and from 2000 to 2008 for pneumonia mortality using national data available. The percentage change in the number of cases and crude and age-adjusted rate was measured. The statistical significance of the annual trends was assessed with the nonparametric trend test for ordered groups. Results: Pneumonia was mainly in children aged 0-4 years and over 65 years old. Morbidity showed a significant upward trend. Deaths were maintained without significant trend observed; analysis by age group went down in all groups except in the 15 to 64 who observed an upward trend. Conclusions: The rising morbidity and overall mortality did not observe any trend, do assume that interventions to improve control of pneumonia are still inadequate, so it is essential to develop future research in Mexican population to identify areas of weakness and health policies needed to observe a favorable change.

Key words: Pneumonia, Mexico, morbidity, mortality, trends.

\section{INTRODUCCIÓN}

Las infecciones de vías respiratorias bajas (IVRB), entre las que se incluye la neumonía, constituyen una de las principales enfermedades en la lista de las primeras 30 causas de mortalidad a nivel mundial. De acuerdo con informes de la Organización Mundial de la Salud, a nivel global las IVRB en el año 2008 ocasionaron la muerte a 3.46 millones de personas, lo que corresponde al $6.1 \%$ del total de muertes para ese año, colocándola en el tercer lugar como causa de muerte después de la enfermedad isquémica del corazón y la enfermedad cerebrovascular. Las cifras para ese mismo rubro en los países de medianos recursos fueron 2.07 millones de muertes, que correspondió al 5.4\% del total de muertes, colocándola en el cuarto lugar. ${ }^{1}$

La tasa global de neumonía adquirida en la comunidad (NAC) varía de 8 a 15 por 1,000 personas por año. En general, hay una variación estacional, con mayor ocurrencia de casos durante los meses de invierno, y es más frecuente en hombres que en mujeres; asimismo, los niños y los adultos mayores padecen esta enfermedad de forma desproporcionada. ${ }^{2}$

La incidencia de neumonía en el mundo varía por país, sexo y edad, lo que afecta en los registros relacionados a la epidemiología, etiología, morbilidad, mortalidad, tasa de resistencia a antibióticos y costo 
económico de la enfermedad. La edad es un determinante importante de la frecuencia de neumonía, como lo demuestra la frecuencia elevada en los menores de 2 años y a partir de los 50 años, aunque la proporción varía dependiendo del país. ${ }^{3}$

El entendimiento y estudio de la morbilidad y mortalidad por neumonía es fundamental para la planeación e implementación de la atención y prevención de estos casos. Las políticas de salud se basan en parte, en conocer la distribución y determinantes de las enfermedades a través del tiempo, esto es importante sobre todo para enfermedades como la neumonía debido a su elevada morbilidad y mortalidad en México y en el mundo.

La planificación sanitaria toma en cuenta la carga relativa de enfermedades y los factores de riesgo que las causan, así como la forma en que dicha carga podría cambiar si se adoptaran políticas e intervenciones de salud específicas. Para medir el impacto de la carga de una enfermedad, es necesario un marco para integrar, validar, analizar y diseminar la información fragmentaria sobre la salud de las poblaciones.

El marco de la carga mundial de morbilidad es una de las tentativas más importantes que se ha hecho para que las medidas de enfermedad de las poblaciones cuenten con datos confiables. ${ }^{4}$

En México, los estudios de epidemiología descriptiva sobre la tendencia de la morbilidad y mortalidad en neumonía en adultos son escasos, por lo que el desarrollo de este trabajo contribuye al conocimiento epidemiológico sobre uno de los principales problemas de salud en México, y tiene implicaciones para la vigilancia epidemiológica y políticas de salud en la población. En este contexto, nuestro objetivo fue describir en población mexicana, las tendencias de morbilidad y mortalidad nacional para neumonía en adultos de 1984 a 2010.

\section{MATERIAL Y MÉTODOS}

Estudio ecológico de series de tiempo retrospectivo de 1984 a 2010, utilizando la información nacional para neumonía disponible en los anuarios de morbilidad del Centro Nacional de Vigilancia Epidemiológica y Control de las Enfermedades (CENAVECE) de la Secretaría de Salud. ${ }^{5}$ En este caso la morbilidad se refiere al número de casos de neumonía por unidad de población registrados por el sistema mencionado durante el período descrito. La información de las estadísticas de mortalidad comprende del año 2000 a 2008 y se obtuvieron del Sistema Nacional de Información en Salud (SINAIS). ${ }^{6}$ En ambos casos se incluyeron los casos definidos como neumonías y bronconeumonías de acuerdo con los códigos de clasificación para neumonía de la décima revisión de la clasificación internacional de las enfermedades $\mathrm{J} 12 \mathrm{a} \mathrm{J} 18$ excepto $\mathrm{J}$ 18.2. Tanto el CENAVECE como el SINAIS cuentan con criterios y procedimientos homogéneos de notificación, por lo que los datos presentados son confiables y representativos de la población mexicana.

Los datos se organizaron y se tabularon para realizar posteriormente el análisis. Hubo variabilidad en la disponibilidad de la información durante el período de estudio señalado, que se reflejará en la sección de análisis y resultados.

La información sobre las tasas en su mayor parte estuvo incluida en los datos obtenidos; no obstante, las tasas específicas por grupo de edad se calcularon con base en la información de la población de México, también disponible en los anuarios de morbilidad a partir de 1990. El cálculo al respecto fue de la siguiente manera: se multiplicó el número de casos (en cada grupo de edad) por 100,000 entre la población total de acuerdo con cada estrato de edad.

El estudio no se sometió a la evaluación del Comité de Ciencia y Ética institucional ya que su desarrollo carecía de riesgo, en virtud de que se utilizaron sólo métodos de investigación documental retrospectiva, sin intervención alguna sobre los individuos que participaron en el estudio.

\section{Análisis}

Se registró y describió toda la información disponible; sin embargo, centramos nuestro análisis y discusión a partir del grupo de 15 años de edad en adelante. Se analizaron las tendencias del número de casos y tasa por año, edad, sexo y por mes conforme a la información que estuviera disponible para cada año en particular. Para la morbilidad se analizó el porcentaje de cambio del número de casos y tasa cruda y ajustada por grupo de edad por año de 1984 a 2010. El porcentaje de cambio también se analizó por sexo para el período 2003-2010.

Se realizó análisis del comportamiento del patrón estacional de la morbilidad por neumonía. Para ello se promediaron los casos de neumonía de cada mes de los años de estudio en que la información estuvo disponible (2003-2010), y se calculó el intervalo de confianza al $95 \%$.

El período de análisis de la mortalidad fue 20002008. De igual manera se midió el porcentaje de cambio del número de casos y tasa cruda y ajustada por grupo de edad entre cada uno de los años del período de estudio.

La significancia estadística de las tendencias anuales tanto para morbilidad y mortalidad se evaluaron 
con prueba de tendencias no paramétrica para grupos ordenados. El análisis se realizó con el paquete estadístico Stata versión 12.0 (StataCorp LP, College Station, TX, USA).

\section{RESULTADOS}

\section{Morbilidad}

Las tendencias tanto del número de casos como de las tasas son muy variables a lo largo del período de estudio, al igual que los cambios relativos de un año a otro, con una tendencia general ascendente y significativa (tabla 1 y figura 1).

Durante el período de estudio se analizó el comportamiento de tres etapas cuya división fue de forma arbitraria: la primera, de 1984 a 1989 que muestra una tendencia ascendente con un incremento relativo en el número de casos y tasa de 63.12 y $47.68 \%$, respectivamente; un segundo período de 1990 a 1999 con una tendencia ascendente y cambios relativos muy pronunciados, más de tres veces que lo observado durante el primer período; y un tercer período de 2000 a 2010 , en donde se advierte una tendencia descendente con cambios relativos para el número de casos y tasa de -23.55 y $-29.75 \%$, respectivamente (tabla 2 ).

Las tasas de neumonía por grupo de edad muestran en todos los casos, una tendencia ascendente con cambios relativos más pronunciados en los adultos de 45 a 64 años y en los de 65 o más años de edad, 68.37 y $153.49 \%$, respectivamente. Asimismo, y aunque la tendencia es ascendente en todos los grupos de edad, ésta fue estadísticamente significativa sólo en los niños menores de un año a cuatro años de edad (tabla 3 y figura 2).

La información disponible respecto al número de casos y tasa por separado para hombres y mujeres demostró lo siguiente: de 2003 a 2010 la tendencia es descendente, tanto en el número de casos como en las tasas, con significancia estadística excepto para el número de casos en los hombres. La tasa mayor del período fue en el año 2003 y la presentaron los hombres con 178.8 casos $/ 100,000$ habitantes, y la menor fue de 142.32 casos/100,000. En el caso de las mujeres, igualmente la tasa mayor fue para el año 2003 con 173.7 casos $/ 100,000$ y la tasa menor de 133.2 casos $/ 100,000$. Se aprecia un ligero predominio del número de casos y tasa en los hombres con respecto a las mujeres (figura 3 ).

El análisis del comportamiento estacional demostró homogeneidad en todos los meses y se observó una distribución normal. El número de casos de los períodos estacionales secuenciales y en el análisis del promedio e intervalo de confianza para cada mes, se documentó que el mes de enero presentó el mayor número de casos/mes 21,157 (IC 95\% de 17,713.43 y 24,600.57). Los meses con el menor número de casos fueron julio y agosto con $8,138.5$ casos/mes (IC 95\% 6,970.95-9,306.05) y 8,244 casos/mes (IC $95 \%$ 6,730.16-9,758.84), respectivamente (figura 4).

En el año 1984, la neumonía ocupó el sexto lugar dentro de las primeras causas de morbilidad nacional, y fue ese mismo año el que reportó la menor tasa del período en estudio. A partir de entonces, los lugares que ha ocupado la morbilidad por neumonía han sido muy variables, ocupando desde el lugar décimo primero hasta el vigésimo, siendo el lugar décimo sexto el más frecuente.

Tabla 1. Morbilidad por neumonía en México (1984-2010).

\begin{tabular}{|c|c|c|c|c|}
\hline Año & $\begin{array}{c}\text { Número de } \\
\text { casos }\end{array}$ & $\begin{array}{l}\text { Porcentaje } \\
\text { de cambio }\end{array}$ & Tasa* $^{*}$ & $\begin{array}{l}\text { Porcentaje } \\
\text { de cambio }\end{array}$ \\
\hline 1984 & 59,182 & & 77.57 & \\
\hline 1985 & 56,255 & -4.94 & 72.8 & -6.15 \\
\hline 1986 & 70,442 & 25.21 & 87.2 & 19.78 \\
\hline 1987 & 88,206 & 25.21 & 108.7 & 24.65 \\
\hline 1988 & 96,382 & 9.26 & 116.51 & 7.18 \\
\hline 1989 & 96,539 & 0.16 & 114.56 & -1.67 \\
\hline 1990 & 87,632 & -9.22 & 102.15 & -10.83 \\
\hline 1991 & 97,268 & 10.99 & 111.47 & 9.12 \\
\hline 1992 & 87,144 & -10.40 & 100.43 & -9.90 \\
\hline 1993 & 85,117 & -2.32 & 96.22 & -4.19 \\
\hline 1994 & 97,356 & 14.37 & 107.98 & 12.22 \\
\hline 1995 & 146,781 & 50.76 & 160.23 & 48.38 \\
\hline 1996 & 169,341 & 15.36 & 181.73 & 13.41 \\
\hline 1997 & 223,469 & 31.96 & 235.9 & 29.80 \\
\hline 1998 & 194,992 & -12.74 & 202.58 & -14.12 \\
\hline 1999 & 232,946 & 19.46 & 237.4 & 17.17 \\
\hline 2000 & 204,886 & -12.04 & 205.7 & -13.35 \\
\hline 2001 & 195,618 & -4.52 & 193.69 & -5.83 \\
\hline 2002 & 214,040 & 9.41 & 209.07 & 8.26 \\
\hline 2003 & 183,671 & -14.18 & 176.2 & -16.02 \\
\hline 2004 & 181,485 & -1.19 & 172.27 & -2.23 \\
\hline 2005 & 172,132 & -5.15 & 161.7 & -6.13 \\
\hline 2006 & 148,464 & -13.74 & 138.07 & -14.61 \\
\hline 2007 & 152,207 & 2.52 & 143.88 & 4.20 \\
\hline 2008 & 146,454 & -3.77 & 137.28 & -4.58 \\
\hline 2009 & 159,049 & 8.59 & 147.88 & 7.72 \\
\hline 2010 & 156,636 & -1.51 & 144.5 & -2.28 \\
\hline z & 3.42 & & 3.09 & \\
\hline$p$ & 0.001 & & 0.002 & \\
\hline
\end{tabular}

*Tasa por 100,000 habitantes.

Fuente: Anuarios de morbilidad del Centro Nacional de Vigilancia Epidemiológica y Control de las Enfermedades de la Secretaría de Salud.

http://www.dgepi.salud.gob.mx/2010/plantilla/inicio_anuarios.html 


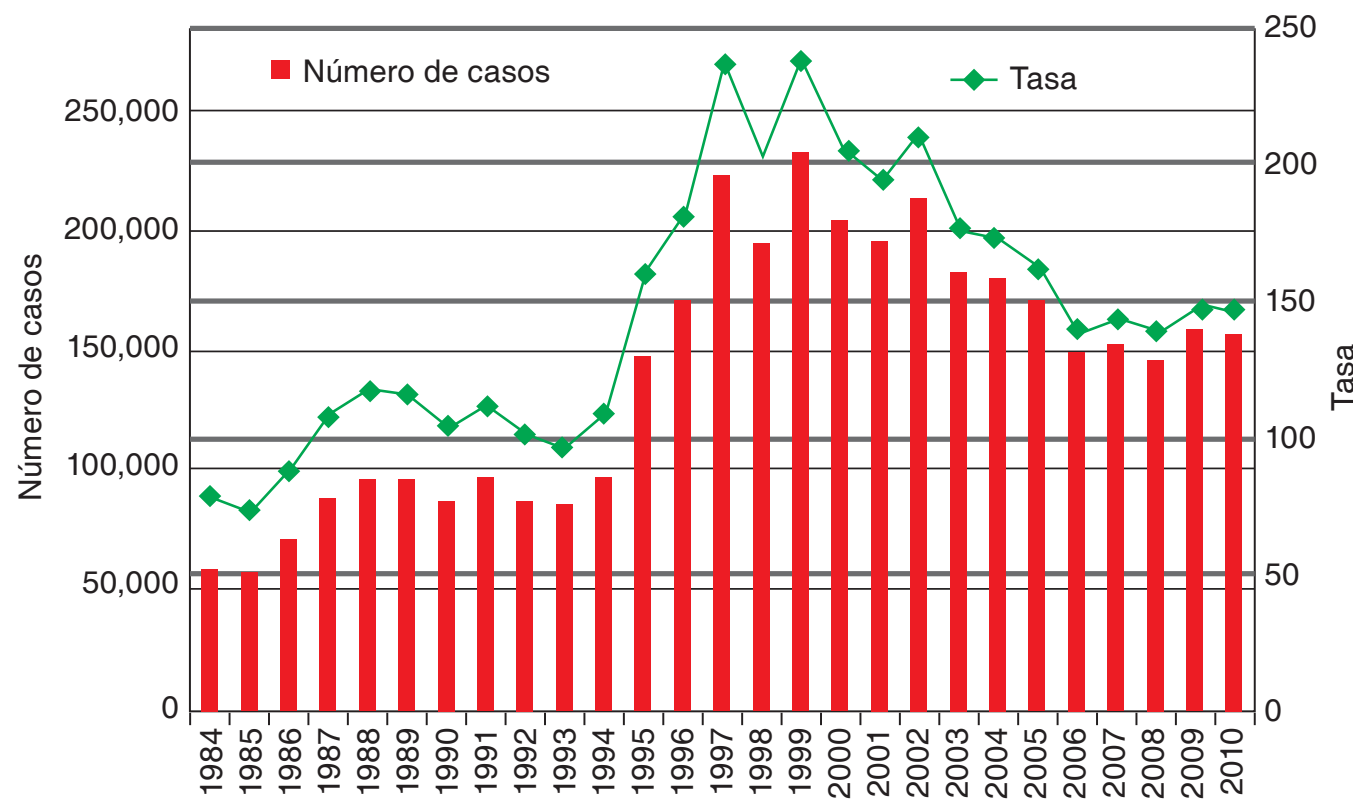

Fuente: Anuarios de morbilidad del Centro Nacional de Vigilancia Epidemiológica y Control de las Enfermedades de la Secretaría de Salud. http://www.dgepi.salud.gob. $\mathrm{mx} / 2010 /$ plantilla/inicio_anuarios.html

Figura 1.

Número de casos y tasa de la incidencia de neumonías en población mexicana por 100,000 habitantes de 1984 a 2010.

Tabla 2. Comportamiento de la morbilidad por neumonía en tres períodos de análisis.

\begin{tabular}{|c|c|c|c|c|c|c|c|c|c|}
\hline & \multicolumn{3}{|c|}{ Período I } & \multicolumn{3}{|c|}{ Período II } & \multicolumn{3}{|c|}{ Período III } \\
\hline & 1984 & 1989 & $\%$ cambio & 1990 & 1999 & \% cambio & 2000 & 2010 & $\%$ cambio \\
\hline $\begin{array}{l}\text { Número de } \\
\text { casos }\end{array}$ & 59,182 & 96,539 & 63.12 & 87,632 & 232,946 & 165.83 & 204,886 & 156,636 & -23.55 \\
\hline Tasa & 77.57 & 114.56 & 47.68 & 102.15 & 237.4 & 132.40 & 205.7 & 144.5 & -29.75 \\
\hline
\end{tabular}

Fuente: Anuarios de morbilidad del Centro Nacional de Vigilancia Epidemiológica y Control de las Enfermedades de la Secretaría de Salud. http://www.dgepi.salud.gob.mx/2010/plantilla/inicio_anuarios.html

\section{Mortalidad}

Durante el período de estudio para este rubro (20002008), los cambios relativos por año fueron variables pero nada notables, lo que se reflejó en el número y tasa de defunciones que se mantuvo sin observarse ninguna tendencia significativa (tabla 4).

El análisis por grupo de edad demostró tendencia descendente en el número de casos y tasa en los menores de un año y hasta los 14 años de edad, con significancia estadística de esta tendencia sólo en el grupo de los menores de un año de edad. El grupo de 15 a 64 años de edad mostró una tendencia ascendente y significativa tanto para el número de casos como para la tasa, con incremento relativo de 24.32 y $8.58 \%$ ( $p<$ $0.05)$, respectivamente. El grupo de los mayores de 65 años presentó una tendencia significativa al alza en el número de casos; la tasa para este mismo grupo de edad demostró una tendencia a la baja con decremento relativo de $-17.21 \%(p>0.05)$ (tabla 5 y figura 5$)$.
La neumonía ocupó el octavo lugar con respecto a las primeras 20 causas de mortalidad en México durante el período de estudio.

\section{DISCUSIÓN}

Hasta donde sabemos, éste es el primer estudio en hacer un análisis detallado de los casos de neumonía en adultos a partir de la información nacional disponible en los anuarios de morbilidad y mortalidad, que emite la Dirección General de Epidemiología y que proporciona información sobre las tendencias de morbilidad y mortalidad nacional con los datos epidemiológicos representativos y disponibles a nivel nacional. Nuestro estudio describe la situación epidemiológica actual respecto a la neumonía, cuyo entendimiento es fundamental para mejorar la planificación e implementación de las medidas de prevención y atención médica en neumonía.

El estudio revela los siguientes hallazgos: la neumonía continúa siendo un problema de salud pública en 
Tabla 3. Tasas de morbilidad de neumonía por grupo de edad en población mexicana (1990-2010).

\begin{tabular}{cccccc}
\hline & \multicolumn{5}{c}{ Grupos de edad } \\
\cline { 2 - 6 } Año & $0-4$ años & $5-14$ años & $15-44$ años & $45-64$ años & $65+$ años \\
\hline 1990 & 432.86 & 62.86 & 36.71 & 74.76 & 204.44 \\
1991 & 491.04 & 65.61 & 38.61 & 82.27 & 232.34 \\
1992 & 400.62 & 53.6 & 35.76 & 80.16 & 207.68 \\
1993 & 419.81 & 48.85 & 30.71 & 72.83 & 233.99 \\
1994 & 495.51 & 54.48 & 34.22 & 75.56 & 242.83 \\
1995 & 636.76 & 78.31 & 57.19 & 146.81 & 467.59 \\
1996 & 805.34 & 77.37 & 54.34 & 147.06 & 559.69 \\
$1997^{*}$ & & & & 649.12 \\
1998 & 849.41 & 99.86 & 65.97 & 174.64 & 709.06 \\
1999 & $1,048.92$ & 116.13 & 72.47 & 202.67 & 603.21 \\
2000 & 898.41 & 101.7 & 64.72 & 192.14 & 585.57 \\
2001 & 830.47 & 98.4 & 63.7 & 185.06 & 588.08 \\
2002 & $1,005.18$ & 105.74 & 59.98 & 176.59 & 538.89 \\
2003 & 784.25 & 94.23 & 57.99 & 160.41 & 463.42 \\
2004 & 945.03 & 79.96 & 44.28 & 130.36 & 509.04 \\
2005 & 797.68 & 82.68 & 46.45 & 135.71 & 423.55 \\
2006 & 713.66 & 72.12 & 37.02 & 112.43 & 469.6 \\
2007 & 717.96 & 70.85 & 38.92 & 115.14 & 489.74 \\
2008 & 642.37 & 61.88 & 39.47 & 118.84 & 464.73 \\
2009 & 600.77 & 81.29 & 56.48 & 143.2 & 153.23 \\
2010 & 648.56 & 70.66 & 44.47 & 125.87 & 1.66 \\
\% cambio & 49.83 & 12.40 & 21.14 & 68.37 & 0.097 \\
z & 1.71 & 1.35 & 1.23 & 1.30 & 0.192 \\
Valor de p & 0.088 & 0.176 & 0.219 & & \\
\hline
\end{tabular}

*Sin información.

Fuente: Anuarios de morbilidad del Centro Nacional de Vigilancia Epidemiológica y Control de las Enfermedades de la Secretaría de Salud. http://www.dgepi.salud.gob.mx/2010/plantilla/inicio_anuarios.html

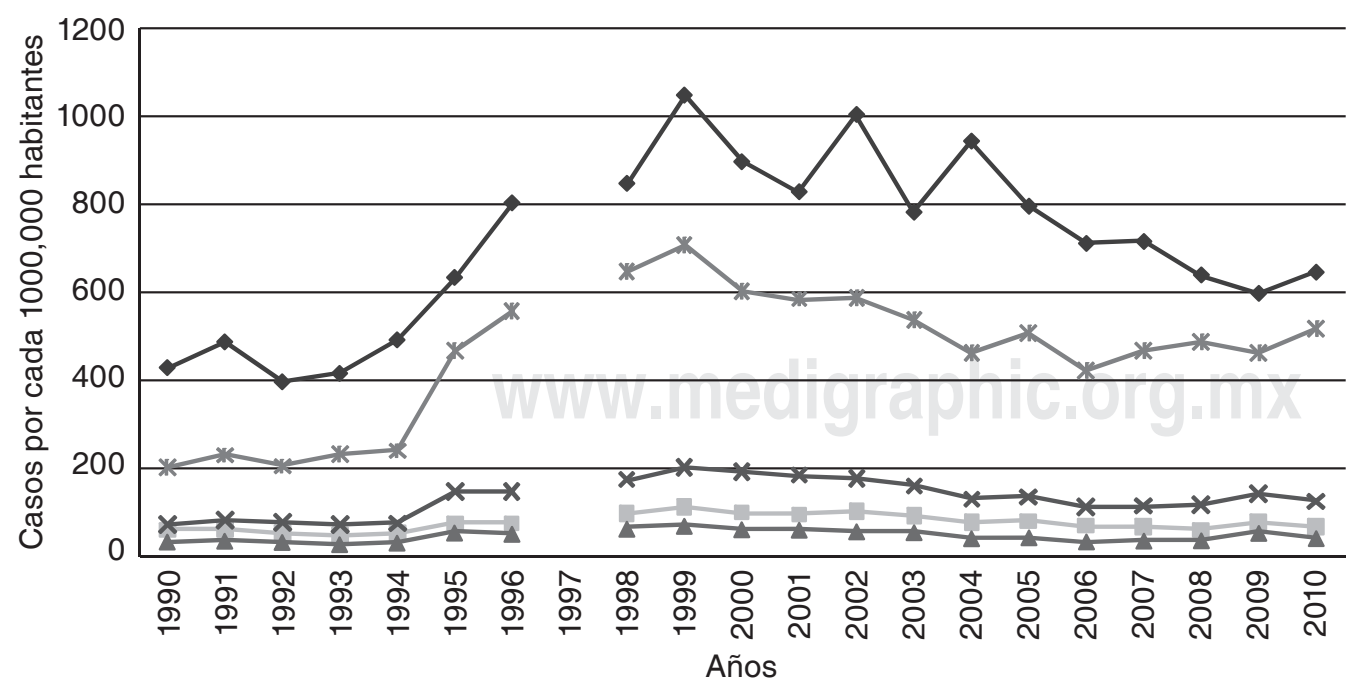

$\rightarrow$ 0-4 años $\quad$ - 5-14 años $\quad$ - 15-44 años $\quad$ * 45-64 años $\quad$ * Más de 65 años
Fuente: Anuarios de morbilidad del Centro Nacional de Vigilancia Epidemiológica y Control de las Enfermedades de la Secretaría de Salud. http://www.dgepi.salud.gob. mx/2010/plantilla/inicio_anuarios.html

\section{Figura 2.}

Tasa de incidencia de neumonía por grupo de edad de 1990 a 2010. 


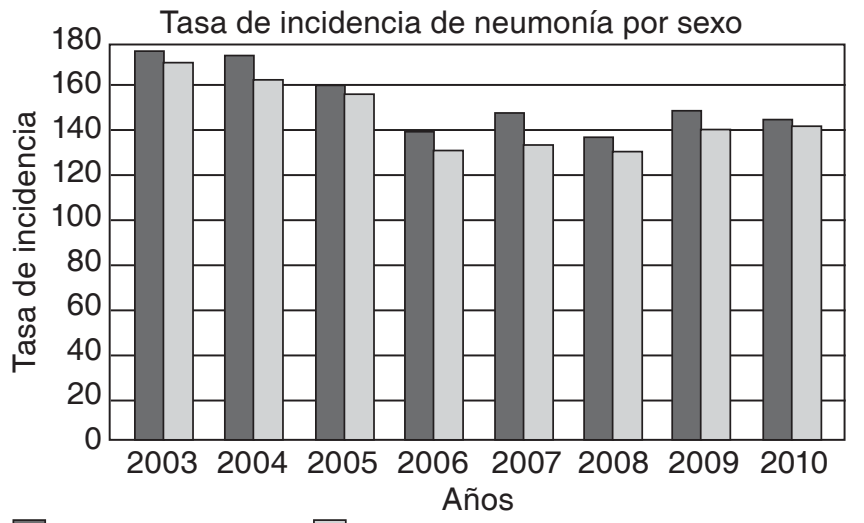

Hombres

Mujeres

Fuente: Anuarios de morbilidad del Centro Nacional de Vigilancia Epidemiológica y Control de las Enfermedades de la Secretaría de Salud. http://www.dgepi.salud.gob.mx/2010/plantilla/inicio_anuarios.html

Figura 3. Tasa de incidencia de neumonía por cada 100,000 habitantes en hombres y mujeres mexicanos de 2003 a 2010.

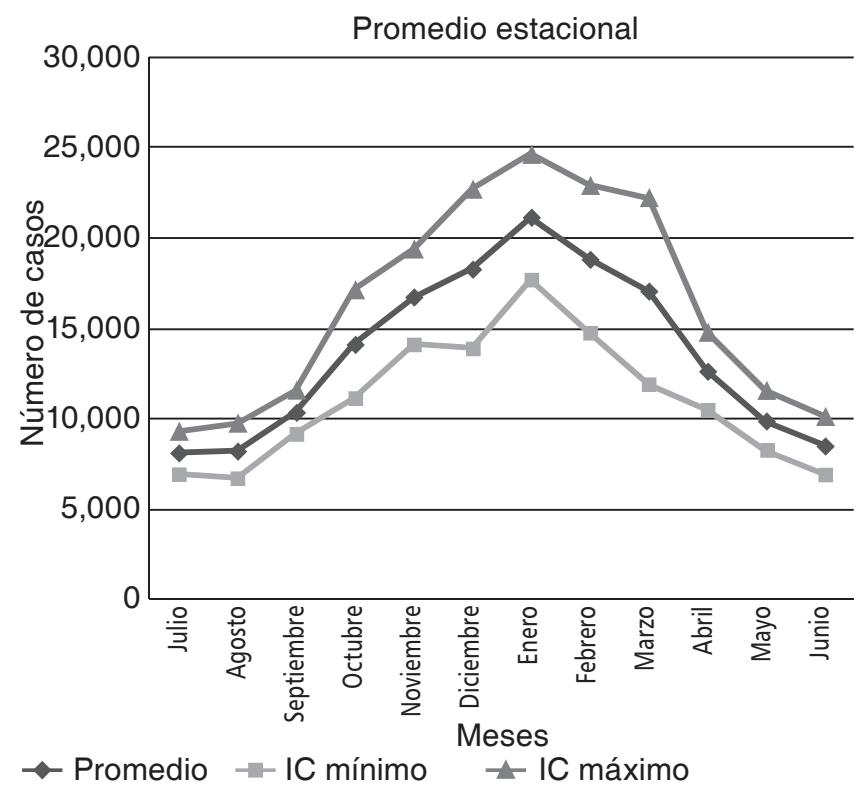

IC: Intervalo de confianza.

Fuente: Anuarios de morbilidad del Centro Nacional de Vigilancia Epidemiológica y Control de las Enfermedades de la Secretaría de Salud. http://www.dgepi.salud.gob.mx/2010/plantilla/inicio_anuarios.html

Figura 4. Patrón estacional del comportamiento de casos de neumonía en población mexicana (2003-2008).

México, afectando a todos los grupos de edad, pero principalmente a los niños de 0-4 años de edad y los adultos de 65 o más años de edad; es un problema ligeramente más frecuente en hombres que en mujeres, esto contrasta con otros informes en donde la diferencia es mucho más notable. ${ }^{7}$ Por otra parte, como se ha
Tabla 4. Defunciones por neumonía (2000-2008).

\begin{tabular}{ccccc}
\hline \multicolumn{5}{c}{ Neumonías } \\
\hline Año & $\begin{array}{c}\text { Número de Porcentaje } \\
\text { casos }\end{array}$ & de cambio & Tasa & $\begin{array}{c}\text { Porcentaje } \\
\text { de cambio }\end{array}$ \\
\hline 2000 & 14,213 & & 14.13 & \\
2001 & 13,101 & -7.82 & 12.87 & -8.92 \\
2002 & 13,662 & 4.28 & 13.26 & 3.03 \\
2003 & 13,738 & 0.55 & 10.07 & -24.42 \\
2004 & 14,215 & 3.47 & 13.5 & 34.06 \\
2005 & 14,979 & 5.37 & 14.4 & 6.66 \\
2006 & 15,180 & 1.34 & 14.5 & 0.69 \\
2007 & 14,589 & -3.89 & 13.8 & -4.82 \\
2008 & 15,096 & 3.47 & 14.8 & 7.24 \\
z & 2.31 & & 1.74 & \\
p & 0.021 & & 0.081 & \\
\hline
\end{tabular}

Fuente: Estadísticas de mortalidad de 2000 a 2008, obtenidas del Sistema Nacional de Información en Salud (SINAIS).

http://sinais.salud.gob.mx/mortalidad/

descrito para otras regiones del mundo, hay un notable componente estacional y muy homogéneo con el mayor número de casos en los meses de diciembre, enero y febrero durante los años 2003 a 2010 (figura 4).

\section{Morbilidad}

En general, durante las casi tres décadas que incluyó el período de estudio para el análisis de morbilidad por neumonía, el número de casos y la tasa de forma global mostraron una tendencia significativa hacia el ascenso; sin embargo, es posible apreciar que de los años 2000 a 2010 la tendencia fue descendente tanto en el número de casos y tasa y que es congruente con el análisis de la tendencia por sexo, que incluyó el período 2003-2010 en el que tanto para los hombres como para las mujeres hubo un porcentaje de cambio negativo y estadísticamente significativo, excepto en el número de casos en el sexo masculino.

La tendencia ascendente en la morbilidad en nuestros resultados es un hallazgo que es concordante con lo descrito por otros estudios de países industrializados, en donde demuestran que la frecuencia de hospitalización por NAC se ha incrementado, argumentando que este comportamiento se debe a cambios demográficos y al incremento en la frecuencia de comorbilidades asociadas como enfermedad cardiovascular, enfermedad pulmonar obstructiva crónica, diabetes y enfermedad neurológica, entre otras, condiciones que predisponen a los pacientes a desarrollar neumonía. ${ }^{8-10}$

Por otra parte, aunque las tendencias son claras, las notables diferencias sobre todo a partir de 1995 con 
Tabla 5. Número de defunciones y tasa/100,000 habitantes por grupos de edad (2000-2008).

\begin{tabular}{|c|c|c|c|c|c|c|c|c|c|c|}
\hline \multirow[b]{3}{*}{ Año } & \multicolumn{9}{|c|}{ Grupos de edad } & \\
\hline & \multicolumn{2}{|c|}{$<1$ año } & \multicolumn{2}{|c|}{1 a 4 años } & \multicolumn{2}{|c|}{5 a 14 años } & \multicolumn{2}{|c|}{15 a 64 años } & \multicolumn{2}{|c|}{ Más de 65 años } \\
\hline & $\mathrm{n}$ & tasa & $n$ & tasa & $n$ & tasa & $\mathrm{n}$ & tasa & $n$ & Tasa \\
\hline 2000 & 3,318 & 137.6 & 668 & 7.35 & 209 & 0.9 & 2,388 & 3.96 & 7,565 & 164.77 \\
\hline 2001 & 2,819 & 123.33 & 575 & 6.31 & 180 & 0.8 & 2,212 & 3.6 & 7,253 & 153.06 \\
\hline 2002 & 3,128 & 143.15 & 662 & 7.27 & 197 & 0.9 & 2,297 & 3.67 & 7,308 & 149.28 \\
\hline 2003 & 2,514 & 119.9 & 626 & 6.96 & 181 & 0.8 & 2,421 & 3.8 & 7,939 & 156.92 \\
\hline 2004 & 2,691 & 132.3 & 677 & 7.7 & 199 & 0.9 & 2,437 & 3.8 & 8,161 & 156.1 \\
\hline 2005 & 2,490 & 123.9 & 572 & 6.8 & 195 & 0.9 & 2,665 & 4 & 8,988 & 166.3 \\
\hline 2006 & 2,281 & 114.6 & 533 & 6.5 & 186 & 0.8 & 2,808 & 4.2 & 9,119 & 163.2 \\
\hline 2007 & 1,969 & 99.9 & 472 & 6 & 149 & 0.7 & 2,810 & 4.1 & 8,161 & 141.1 \\
\hline 2008 & 1,719 & 87.9 & 448 & 5.8 & 145 & 0.7 & 2,969 & 4.3 & 8,161 & 136.4 \\
\hline $\begin{array}{c}\% \text { de } \\
\text { cambio }\end{array}$ & -48.19 & -36.11 & -32.93 & -21.08 & -30.62 & -22.22 & 24.32 & 8.58 & 7.87 & -17.21 \\
\hline z & -2.73 & -2.22 & -2.22 & -1.79 & -1.74 & -1.76 & 2.69 & 2.30 & 2.16 & -1.08 \\
\hline$p$ & 0.006 & 0.027 & 0.027 & 0.073 & 0.081 & 0.078 & 0.007 & 0.022 & 0.031 & 0.278 \\
\hline
\end{tabular}

Fuente: Estadísticas de mortalidad de 2000 a 2008, obtenidas del Sistema Nacional de Información en Salud (SINAIS). http://sinais.salud.gob.mx/mortalidad/

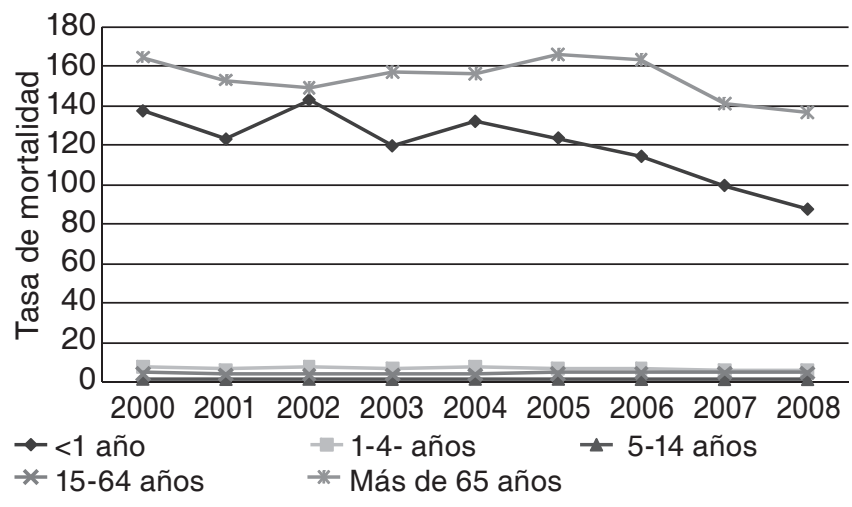

Fuente: Estadísticas de mortalidad de 2000 a 2008, obtenidas del Sistema Nacional de Información en Salud (SINAIS). http://sinais.salud.gob.mx/mortalidad/

Figura 5. Tasa de mortalidad por neumonía por grupo de edad de 2000 a 2008.

tendencia ascendente y del año 2000 con tendencia descendente, se pueden deber a la calidad de la atención de los diferentes servicios médicos, acceso de la población al servicio médico, factores sociales y densidad de población, ${ }^{11}$ y cuyo análisis de estas situaciones queda fuera del alcance de este estudio debido a su diseño.

\section{Mortalidad}

La mortalidad cruda en la población total no mostró ninguna tendencia ni en el número de casos ni en las tasas. El análisis por grupos de edad demostró que en los niños menores de un año de edad, la tendencia fue descendente y estadísticamente significativa, tanto para el número de casos como para la tasa; en contraste, el grupo de 15 a 64 años mostró una tendencia al ascenso estadísticamente significativa. La tasa de neumonía en el grupo de los mayores de 65 años de edad presentó un ligero descenso estadísticamente significativo. Aunque con los datos disponibles y diseño del estudio, no es factible saber con certeza el motivo de la ausencia de disminución de la mortalidad cruda en la población total, ésta tiene varias explicaciones posibles. De acuerdo con la evidencia médica, una parte se podría deber a la falta de apego a las guías clínicas para neumonía, particularmente en lo referente al esquema de antibiótico apropiado y a la cobertura de vacunación contra influenza y neumococo. Estudios previos han demostrado que la vacunación contra el neumococo tiene un impacto muy bajo sobre la incidencia de neumonía; sin embargo, hay evidencia contundente que disminuye el riesgo de bacteriemia y enfermedad invasiva por neumococo, ambas circunstancias aumentan el riesgo de muerte, por lo que el haber recibido la vacuna impactó sobre el riesgo de muerte que disminuyó ostensiblemente. ${ }^{12}$ En este mismo sentido, en un estudio de pacientes hospitalizados por neumonía se documentó una asociación entre la disminución del riesgo de mortalidad y el haber recibido vacuna de la influenza. ${ }^{13}$

La vacunación contra el neumococo y la influenza es una medida fundamental para la prevención de la 
neumonía y que también repercute en el desenlace de la misma una vez que el paciente está afectado. La vacunación en este caso, generalmente va dirigida a los grupos más vulnerables como son los niños menores de 2 años y los adultos de 60 años o más, esto podría explicar el incremento relativo en la mortalidad de 24.32 y $8.58 \%$ en el número de casos y tasa, respectivamente, que se documentó en nuestro estudio en el grupo de 15 a 64 años de edad, debido a que es un grupo en el que generalmente no van dirigidas las medidas de prevención de neumonía. A favor de este argumento está un estudio de casos y controles realizado en México durante la reciente pandemia de influenza A (H1N1) en el año 2009, en la que se demostró que el padecer influenza $A$ (H1N1) se asoció independientemente con el antecedente de vacunación contra influenza (OR 0.27 95\% IC 0.11-0.66), con una eficacia de la vacuna de $73 \%$ (IC 95\% 34-89\%), y se concluye que es muy probable que la vacuna trivalente inactivada administrada durante 2008-2009 confirió protección principalmente contra formas graves de la enfermedad. ${ }^{14}$

En este mismo contexto, otro estudio en pacientes hospitalizados describió resultados opuestos a los nuestros, debido a que la mortalidad por neumonía ajustada por edad y sexo, disminuyó de $8.9 \%$ a $4.1 \% .^{15}$ Congruente con este estudio, un análisis de datos administrativos sobre la tendencia de mortalidad por neumonía en adultos hospitalizados como ambulatorios de 80 años o más, en el período 1987-2005 demostró que la mortalidad disminuyó significativamente en este grupo de edad, y esta disminución fue consistente con el apego a los criterios de tratamiento antibiótico de las guías para neumonía y a la vacunación para neumococo e influenza. ${ }^{10}$ En nuestro estudio, en los mayores de 65 años de edad también demostramos una reducción relativa no significativa de $-17.21 \%$, aunque por el diseño del estudio, no es posible documentar cuáles son los factores asociados a dicha disminución.

Un estudio en México sobre la cobertura nacional de vacunación contra influenza y neumococo en el adulto mayor de 60 años, demostró que ésta fue de $56.5 \%( \pm 0.97)$ para influenza y para neumococo $44.3 \%$ $( \pm 0.98),{ }^{16}$ y aunque estos resultados representan un avance significativo, es necesario implementar políticas de salud y estrategias para incrementar la cobertura de vacunación en este grupo de edad. Está demostrado el impacto de la vacuna contra influenza sobre la reducción en la mortalidad por neumonía e influenza en menores de dos años y mayores de 65 años de acuerdo con los resultados de un estudio realizado en México entre 1990-2005. ${ }^{17}$ Una tendencia semejante la documentamos en el presente estudio en ambos grupos de edad.
El análisis separado de la mortalidad por grupos de edad demuestra que los grupos más afectados son los menores de 1 a 4 años de edad y los mayores de 65 años; y contrario a lo que ocurre con la tasa de morbilidad por neumonía, la mortalidad fue 1.2 veces más frecuente en los mayores de 65 años de edad comparado con los menores de uno a cuatro años.

\section{Limitaciones del estudio}

Entre éstas resalta su diseño retrospectivo y problemas asociados con la calidad de los registros de información y su codificación. En particular, que los casos de neumonía que se describen no permiten separar los casos de NAC de los otros tipos de neumonía como la nosocomial, asociada al ventilador, a cuidados de la salud o de la neumonía en el paciente inmunocomprometido. Respecto a la mortalidad, puede haber problemas con el denominador seleccionado y con el retraso en el registro de las defunciones; asimismo, con las proyecciones de la población realizadas por la Comisión Nacional de Población que podrían subestimar las tasas de muerte durante el quinquenio inmediato posterior. Por otra parte, no fue posible identificar los factores relacionados al comportamiento de las tendencias de la morbilidad y mortalidad observadas, dado que no disponemos de información sobre la frecuencia y tipo de comorbilidades, tabaquismo y cobertura de vacunación, que desde luego constituyen factores de gran importancia que inciden en estos indicadores epidemiológicos. ${ }^{18,19}$

\section{CONCLUSIONES}

La morbilidad en ascenso y la mortalidad general que no observa alguna tendencia, hace suponer que las intervenciones para mejorar el control de esta enfermedad aún son insuficientes, por lo que es necesario fortalecer las medidas de prevención de neumonía mediante la vacunación, así como el desarrollo de investigación futura en población mexicana para identificar las áreas de debilidad y las políticas de salud necesarias para observar un cambio favorable.

\section{REFERENCIAS}

1. World Health Organization. 2014. The 10 leading causes of death in the world, 2012. Fact sheet No. 310. Updated May 2014. Access date: August 14, 2012. Available from: http://www.who.int/mediacentre/factsheets/fs310/en/ index.html

2. Welte T, Köhnlein T. Global and local epidemiology of community-acquired pneumonia: the experience of 
the CAPNETZ Network. Semin Respir Crit Care Med 2009;30(2):127-135. doi: 10.1055/s-0029-1202941.

3. Jokinen $\mathrm{C}$, Heiskanen $\mathrm{L}$, Juvonen $\mathrm{H}$, et al. Incidencie of community-acquired pneumonia in the population of four municipalities in Eastern Finland. Am J Epidemiol 1993;137(3):977-988.

4. López $A D$, Mathers $C D$, Ezzati M, Jamison DT, Murray CJL. Global burden of the disease and risk factors. Capítulo 1: La medición de la carga mundial de morbilidad y de los factores de riesgo, 1990-2001. OMS. 1-15.

5. Secretaría de Salud. Anuarios de morbilidad de la Dirección General de Epidemiología. Fecha de consulta: 20 de septiembre, 2012. Accesible en: http://www.dgepi. salud.gob.mx/2010/plantilla/inicio_anuarios.html

6. Secretaría de Salud. Sistema Nacional de Información en Salud. Mortalidad. Información 2000-2008. Fecha de consulta: 30 de septiembre, 2012. Accesible en: http:// sinais.salud.gob.mx/mortalidad/

7. American Lung Association Research and Program Services Epidemiology and Statistics Unit April 2010. Trends in Pneumonia and Influenza Morbidity and Mortality.

8. Trotter CL, Stuart JM, George R, Miller E. Increasing hospital admissions for pneumonia, England. Emerg Infect Dis 2008;14(5):727-733. doi: 10.3201/eid1405.071011.

9. Fry AM, Shay DK, Holman RC, Curns AT, Anderson LJ. Trends in hospitalizations for pneumonia among persons aged 65 years or older in the United States, 1988-2002. JAMA 2005;294(21):2712-2719.

10. Ruhnke GW, Coca-Perraillon M, Kitch BT, Cutler DM. Marked reduction in 30-day mortality among elderly patients with community-acquired pneumonia. Am J Med 2011;124(2):171-178.e1. doi: 10.1016/j.amjmed.2010.08.019.

11. Fine MJ, Auble TE, Tealy DM, et al. A prediction rule to identifiy low-risk patients with community-acquired pneumonia. N Engl J Med 1997;336(4):243-250.

12. Vila-Córcoles A, Ochoa-Gondar O, Hospital I, et al; EVAN Study Group. Protective effects of the 23-valente pneumococcal polysacharide vaccine in the elderly population: the EVAN-65 study. Clin Infect Dis 2006;43(7):860868.
13. Spaude KA, Abrutyn E, Kirchner C, Kim A, Daley J, Fisman DN. Influenza vaccination and risk of mortality among adults hospitalized with community-acquired pneumonia. Arch Intern Med 2007;167(1):53-59.

14. García-García L, Valdespino-Gómez JL, Lazcano-Ponce E, et al. Partial protection of seasonal trivalent inactivated vaccine against novel pandemic influenza A/H1N1 2009: case-control study en Mexico City. BMJ 2009;339:b3928. doi: 10.1136/bmj.b3928.

15. Ruhnke GW, Coca-Perraillon M, Kitch BT, Cutler DM. Trends in mortality and medical spending in patients hospitalized for community-acquired pneumonia: 19932005. Med Care 2010;48(12):1111-116. doi: 10.1097/ MLR.0b013e3181f38006.

16. Trejo-Valdivia B, Mendoza-Alvarado LR, Palma-Coca O, Hernández-Ávila M, Téllez-Rojo Solís MM. Encuesta Nacional de Cobertura de Vacunación (influenza, neumococo y tétanos) en adultos mayores de 60 años en México. Salud Pública Mex 2012;54(1):39-46.

17. Kuri-Morales $P$, Galván F, Cravioto P, Zárraga-Rosas LA, Tapia-Conyer R. Mortalidad en México por influenza y neumonía (1990-2005). Salud Pública Mex 2006;48(5):379-384.

18. Fine MJ, Smith MA, Carson CA, et al. Prognosis and outcomes of patients with community-acquired pneumonia. A meta-analysis. JAMA 1996;275(2):134-141.

19. Feikin DR, Schuchat A, Kolczak M, et al. Mortality from invasive pneumococcal pneumonia in the era of antibiotic resistance, 1995-1997. Am J Public Health 2000;90(2):223-229.

\section{$\triangle$ Correspondencia:}

Dra. Renata Báez-Saldaña

Jefe del Servicio Clínico 3, Instituto Nacional de Enfermedades Respiratorias Ismael Cosío Villegas. Calzada de Tlalpan Núm. 4502, Colonia Sección XVI. 14080, México, D.F.

Tel: (52) 5554871700 , ext. 5284

Correo electrónico: baezrd@unam.mx

Los autores declaran no tener conflicto de intereses. 